\title{
A Case of Retroperitoneal Primary Neuroblastoma that Developed in Adulthood
}

Shunsuke Watanabe*, Fujio Hara, Yasuhiro Kondo, Tatsuya Suzuki, Toshihiro Yasui, Naoko Uga and Atuki Naoe

Department of Pediatric Surgery, Fujita Health University, Japan

\begin{abstract}
Neuroblastoma is a solid malignant tumor found in young children period, but it may also develop in adulthood. Adult neuroblastoma is rare in Japan (incidence of at least $1 / 100$ million). Here, we report on an adult with retroperitoneal primary neuroblastoma. A 32-year-old woman presented with a chief complaint of abdominal tumor diagnosed as neuroblastoma on needle biopsy. The tumor markers for neuroblastoma were $32.6 \mathrm{ng} / \mathrm{ml}$ for neuron specific enolase, $30.1 \mu \mathrm{g} / \mathrm{mg} \mathrm{Cr}$ for urine vanillylmandelic acid and $42.3 \mu \mathrm{g} / \mathrm{mg} \mathrm{Cr}$ for urine homovanillic acid. Imaging showed metastasis to the mediastinal lymph nodes, but bone metastases were not found. The International Neuroblastoma Risk Group Staging System classification for this neuroblastoma was stage M. The tumor involved the left renal artery and aorta. The case was positive for image defined risk factors. Therefore, the patient received three courses of cyclophosphamide, pirarubicin, vincristine, and cisplatin. Tumor shrinkage was noted. We recommended continued chemotherapy, and bone marrow transplantation, but the patient refused further chemotherapy due to the serious side effects of chemotherapy. Tumor extirpation was judged possible by resection of the left renal tumor using a retroperitoneal mirror in combination. The excised tumor was more ganglion-like than at biopsy, and a stroma-rich portion was recognized, which was thought to be maturation due to chemotherapy. MYCN gene amplification was absent. Postoperatively, radiotherapy of the tumor bed and mediastinal lymph nodes was performed and the patient was followed-up. Many adults refuse chemotherapy, which makes sufficient treatment difficult. In adults, even when MYCN gene amplification is not allowed, sufficient observation is necessary since prognosis is the poor.
\end{abstract}

Keywords: Neuroblastoma; Adult; Retroperitoneal; Retroperitoneal mirror

\section{Introduction}

Neuroblastoma is a solid malignant tumor typically seen in children, but rare adult onset cases exist. In general, the prognosis of adults with neuroblastoma is poor and, to our knowledge, no standard treatment has been established. Here, we report on an adult with primary peritoneal neuroblastoma and review the literature.

\section{Case Report}

A 32-year-old woman presented to our hospital with a chief complaint of an abdominal mass, which had been diagnosed as neuroblastoma by needle biopsy elsewhere two months previously. In the hospital, a blood test revealed $32.6 \mathrm{ng} / \mathrm{mL}$ neuron-specific enolase (NSE), $30.1 \mu \mathrm{g} / \mathrm{mg} \bullet \mathrm{Cr}$ vanillylmandelic acid, and $42.3 \mu \mathrm{g} / \mathrm{mg} \cdot \mathrm{Cr}$ homovanillic acid in the urinalysis.

Computerized tomography (CT) showed a $15 \mathrm{~cm}$ diameter tumor, which was partially calcified (Figure 1a).

Contrast CT revealed that the tumor involved the left renal artery and surrounded more than half the circumference of the abdominal aorta positive for image defined risk factors [IDRFs]; (Figure 1b).

Metaiodobenzylguanidine scintigraphy showed accumulation in the primary tumor but not in metastases. However, on positron emission tomography (PET), metastasis was suspected in the lower esophageal and mediastinal lymph nodes in addition to the accumulation noted in the primary lesion (Figure 1c).

Based on these findings, the disease was classified as neuroblastoma stage $\mathrm{M}$ according to the International Neuroblastoma Risk Group Staging System. Chemotherapy was initiated with reference to the children's guidelines with the regimen 05 A 3 (cyclophosphamide, $1200 \mathrm{mg} / \mathrm{m}^{2}$; pirarubicin, $40 \mathrm{mg} / \mathrm{m}^{2}$; vincristine, $1.5 \mathrm{mg} / \mathrm{m}^{2}$; cisplatin, $18 \mathrm{mg} / \mathrm{m}^{2}$ ) in three courses, with $75 \%$ of the dose administered in the first course and $100 \%$ in the second and third courses. In addition, one course of ICE chemotherapy (ifosfamide, $1800 \mathrm{mg} / \mathrm{m}^{2}$; carboplatin, 400 $\mathrm{mg} / \mathrm{m}^{2}$; and etoposide, $100 \mathrm{mg} / \mathrm{m}^{2}$ ) was administered (Table 1).

Chemotherapy resulted in shrinkage of the primary tumor, with less than half of the aorta encircled, but the renal artery was surrounded and IDRF was positive.

Therefore, we recommended continued chemotherapy and bone marrow transplantation, but the patient refused further chemotherapy. We thought that extirpation of the kidney tumor was possible and surgery was performed.

Surgery was combined with the use of a retroperitoneal mirror. First, four ports were inserted from the left abdomen to the retro peritoneum with the patient in the right half lateral decubitus position. The dorsal side of the tumor was peeled and advanced under the retroperitoneal cavity.

The artery branching into the tumor and retroperitoneal mucosa was dissected. Next, the patient was placed in the supine position and the abdomen was opened via a midline incision. The retroperitoneal cavity was approached from outside of the colon.

*Corresponding author: Shunsuke Watanabe, Department of Paediatric Surgery Fujita Health University, Japan, Tel: 0562-93-9247, 0562-93-2323; Fax: 0562-931951; E-mail: watanabe@fujita-hu.ac.jp

Received February 05, 2018; Accepted February 16, 2018; Published February 20, 2018

Citation: Watanabe S, Hara F, Kondo Y, Suzuki T, Yasui T, et al. (2018) A Case of Retroperitoneal Primary Neuroblastoma that Developed in Adulthood. J of Tumor Res \& Reports 3: 116

Copyright: @ 2018 Watanabe S, et al. This is an open-access article distributed under the terms of the Creative Commons Attribution License, which permits unrestricted use, distribution, and reproduction in any medium, provided the original author and source are credited. 
Citation: Watanabe S, Hara F, Kondo Y, Suzuki T, Yasui T, et al. (2018) A Case of Retroperitoneal Primary Neuroblastoma that Developed in Adulthood. J of Tumor Res \& Reports 3: 116.

The tumor was detached and the ovarian arteries and veins and the ureter were ligated and detached. Subsequently, the renal vein was isolated, ligated, and detached. Finally, we peeled the tumor from the aorta; the adhesion was strong and the boundary was unclear, but we were finally able to detach the tumor from the aorta.

Two renal arteries were ligated and dissected and the complicated renal tumor was excised. The specimen, including the tumor and the left kidney, was $22 \times 12 \times 9 \mathrm{~cm}$ in size and weighed $998 \mathrm{~g}$ (Figure 2a). The operative time was 16 hours and 11 minutes and the estimated blood loss was $2980 \mathrm{ml}$, which mostly occurred after laparotomy (Figure 2a).

Histological findings at needle biopsy revealed a heterozygous tumor with a high nuclear cytoplasmic ratio. Immuno histologically, the tumor was positive for CD56 but negative for NF and S100. The diagnosis was poorly differentiated neuroblastoma.

After chemotherapy, histology revealed more ganglion-like cells and an increased stroma-rich area than noted at the biopsy. Immuno histologically, the tumor was NSE- and CD56-positive, but S100negative. However, NF was partially positive (Figure $2 b$ ).

In addition, the mitosis-karyorrhexis index (MKI) was less than $2 \%$, and MYCN gene amplification and 1-bp deletion were not observed (Figure 2b).

Postoperatively, transient platelet reduction and mild renal dysfunction were observed but lightened. Tumor markers also declined

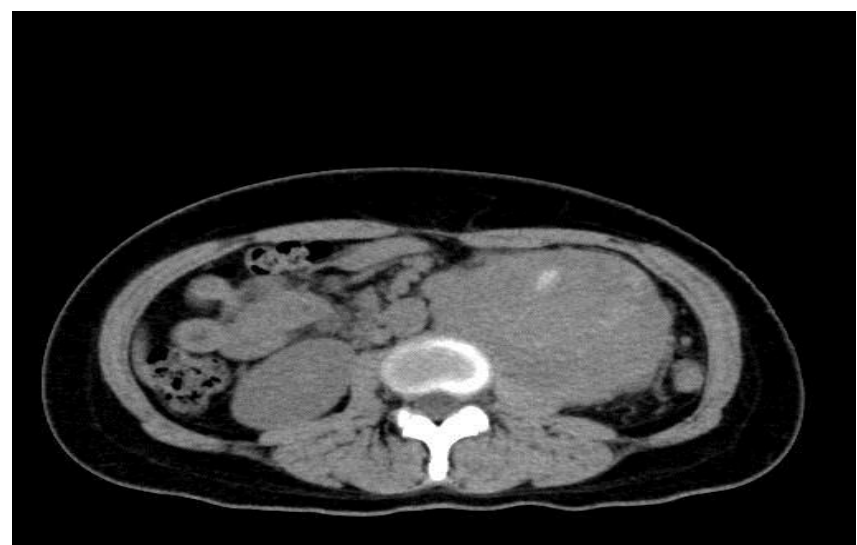

Figure 1a: Computerized tomography (CT) showed a $15 \mathrm{~cm}$ diameter tumor, which was partially calcified.

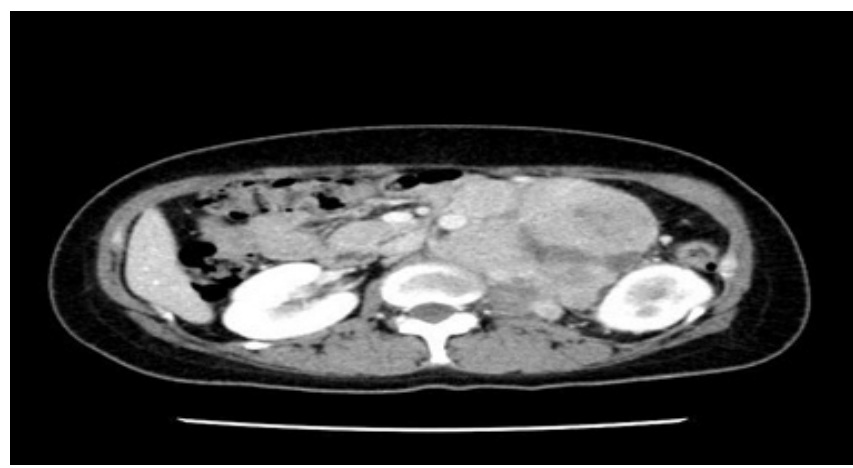

Figure 1b: Contrast CT revealed that the tumor involved the left renal artery and surrounded more than half the circumference of the abdominal aorta.
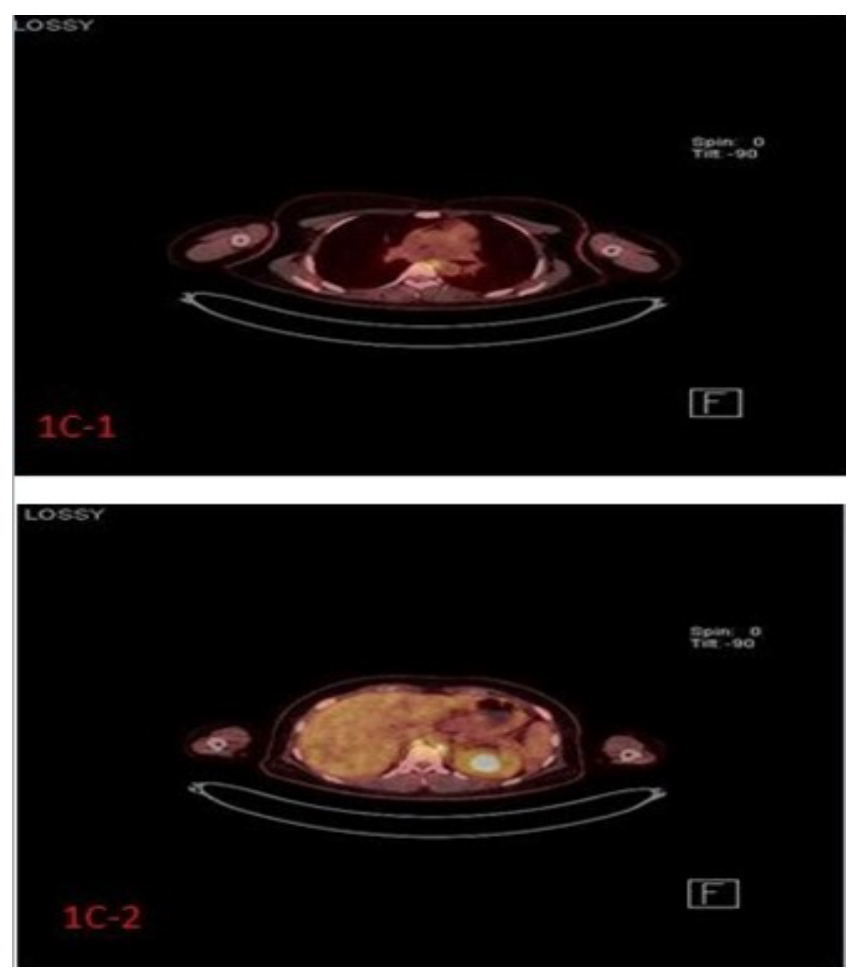

Figure 1c: On positron emission tomography (PET), metastasis was suspected in the lower esophageal and mediastinal lymph nodes in addition to the accumulation noted in the primary lesion.

after removal (Table 2). Postoperative chemotherapy was scheduled due to stage IV disease (International Neuroblastoma Staging System classification), but the patient refused chemotherapy due to the serious side effects of chemotherapy. However, radiation therapy (36 Gy) was administered to the tumor bed and mediastinal lymph nodes. Currently, five months after the end of treatment, accumulation in the lower esophageal and mediastinal lymph nodes was confirmed by PET CT without an increase in the tumor markers (Table 2).

\section{Discussion}

Neuroblastoma is a solid malignant tumor derived from the neural crest and is seen in infancy. It is thought to be due to an abnormality in the process of differentiation and maturation of neuroblast forming the adrenal medulla and the sympathetic ganglia. Neuroblastoma is classified into three basic forms, neuroblastoma, ganglioneuroblastoma, and ganglionoma, because the neuroblastoma histology differs greatly depending on its degree of differentiation. Therefore, the degree of differentiation of tumor cells and the International Neuroblastoma Pathology Classification (INPC), with MKI as an index of cell division/nuclear decay and patient age, are used for the international classification of pathological tissues. In this case, the pathological histology at biopsy was poorly differentiated neuroblastoma, which carries a poor prognosis due to the INPC classification. However, the histopathological imaging at operation revealed many ganglion-like, stroma-rich cells, and partly positive NF and differentiation progressing in some tissues. It was thought that maturation occurred due to the influence of chemotherapy.

As mentioned above, neuroblastoma is a solid malignancy typically 
Citation: Watanabe S, Hara F, Kondo Y, Suzuki T, Yasui T, et al. (2018) A Case of Retroperitoneal Primary Neuroblastoma that Developed in Adulthood. J of Tumor Res \& Reports 3: 116.

Page 3 of 5
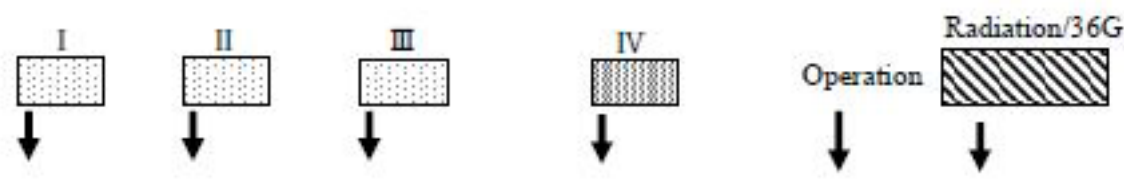

$\mu \mathrm{g} / \mathrm{mg} \cdot \mathrm{Cr}$

Chemotherapy I : 05A3(CPA, THP, VCR, CDDP) dose $75 \%$ Chemotherapy II, III: 05A3(CPA, THP, VCR, CDDP)dose $100 \%$

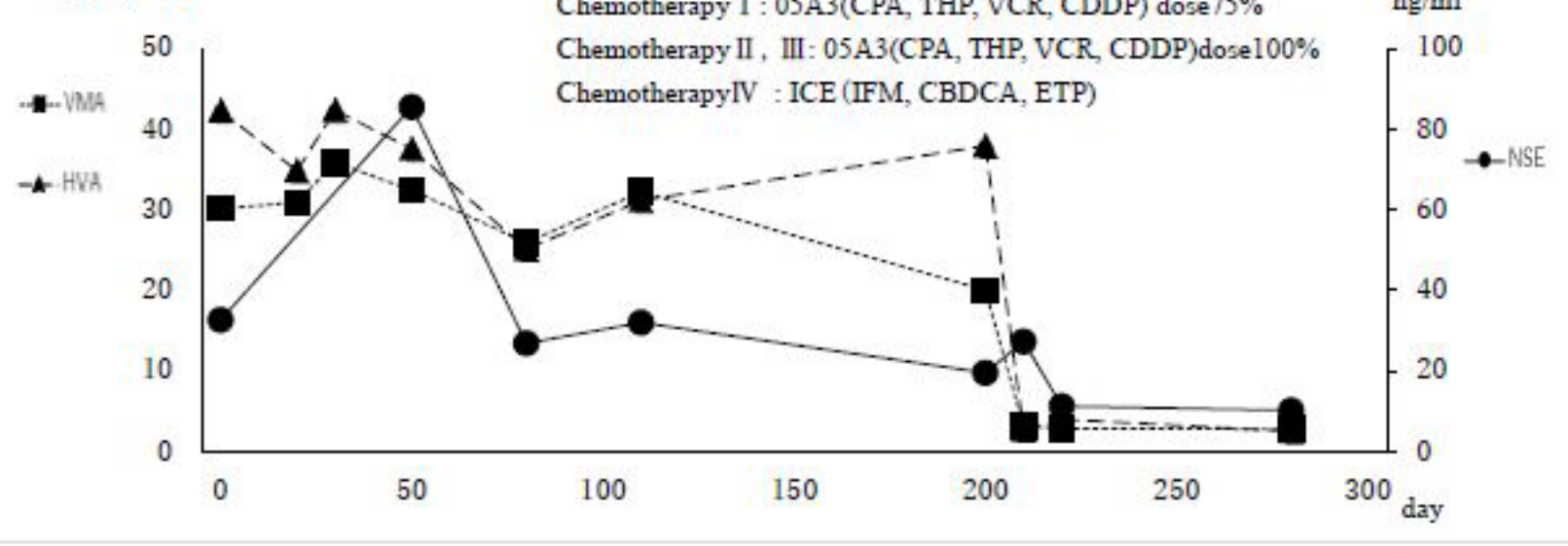

Table 1: Treatment course.

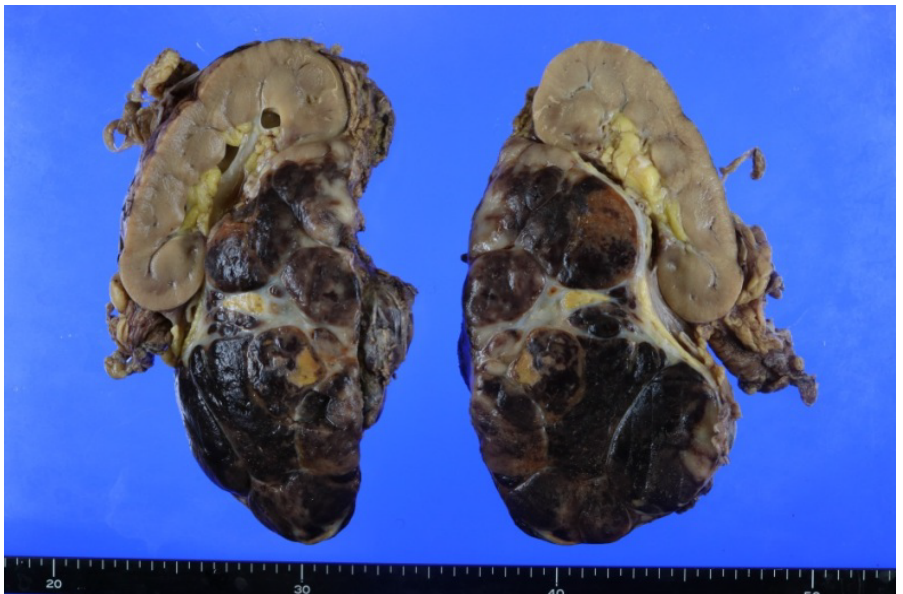

Figure 2a: Histological findings at needle biopsy revealed a heterozygous tumor with a high nuclear cytoplasmic ratio. Immuno histologically, the tumor was positive for CD56 but negative for NF and S100. The diagnosis was poorly differentiated neuroblastoma.
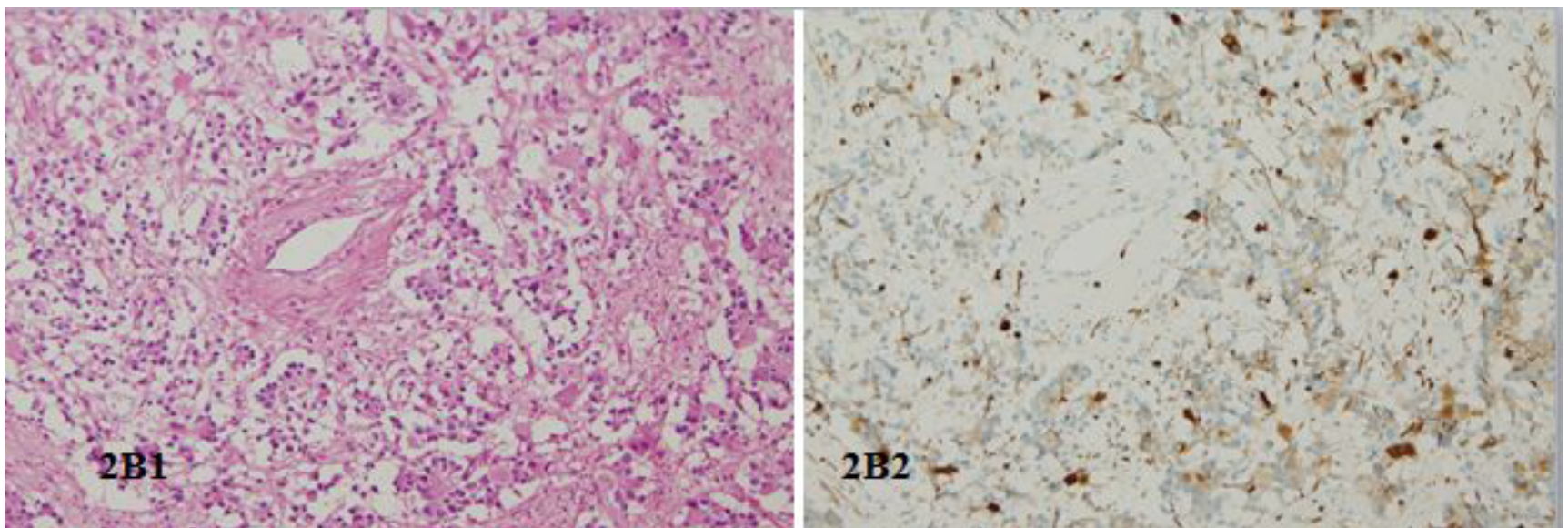

Figure 2b: Histological findings after chemotherapy were more ganglion-like cells and anincreased stroma-rich area than noted at the biopsy. Immunohistologically, the tumor was NSE- and CD56-positive, but S100-negative. However, NF was partially positive. 
Citation: Watanabe S, Hara F, Kondo Y, Suzuki T, Yasui T, et al. (2018) A Case of Retroperitoneal Primary Neuroblastoma that Developed in Adulthood. J of Tumor Res \& Reports 3: 116.

Page 4 of 5

\begin{tabular}{|c|c|c|c|c|c|c|c|c|}
\hline Patient no. & Age & Sex & Stage & Surgery & Chemotherapy & Radiation & Outcome & Survival (mo) \\
\hline 1 & 28 & $M$ & II & Yes & CPM, VCR & No & Alive & 18 \\
\hline 2 & 64 & $\mathrm{~F}$ & IV & Yes & CPM, VCR & No & Dead & 11 \\
\hline 3 & 20 & $\mathrm{~F}$ & IV & Yes & Unknown & Unknown & Unknown & Unknown \\
\hline 4 & 30 & $\mathrm{~F}$ & III & Yes & CPM, VCR, ADM, DTIC & No & Alive & 10 \\
\hline 5 & 43 & $\mathrm{~F}$ & IV & No & No & No & Dead & 7 \\
\hline 6 & 56 & $\mathrm{~F}$ & III & Yes & CHP, THP, ADM, CDDP, VP-16, IFM & No & Dead & 12 \\
\hline 7 & 43 & $\mathrm{~F}$ & III & No & Unknown & No & Dead & 12 \\
\hline 8 & 34 & M & IV & Yes & CDDP, ETP, BLM, ADM, CPA, VCR, DTIC, PBSCT & Yes & Dead & 17 \\
\hline 9 & 26 & M & III & Yes & CPM, VP-16, THP-ADM, CDDP & Yes & Alive & 70 \\
\hline 10 & 34 & $\mathrm{~F}$ & 1 & No & Yes & Yes & Dead & 11 \\
\hline 11 & 74 & M & IV & No & No & Yes & Dead & 3 \\
\hline 12 & 36 & $\mathrm{M}$ & 1 & Yes & Yes & No & Alive & 84 \\
\hline 13 & 24 & $\mathrm{~F}$ & III & Yes & Yes & Yes & Alive & 72 \\
\hline 14 & 27 & M & IV & Yes & VCR, ACT-D, IFM, DXR, CDDP, VP-16 & Yes & Dead & 41 \\
\hline 15 & 69 & $\mathrm{M}$ & IV & No & No & No & Dead & 1 \\
\hline 16 & 32 & $\mathrm{~F}$ & IV & Yes & CPA, THP, VCR, CDDP, IFM, VP-16 & Yes & Alive & 5 \\
\hline
\end{tabular}

Table 2: Adult retroperitoneal primary neuroblastoma in Japan, including our case.

seen in childhood, and adult onset cases are very rare. In addition, the pathology, treatment, and prognosis of adult neuroblastoma are unclear. The incidence of adult neuroblastoma has been reported as being one in one billion people [1], and the age of onset has also been widely reported (16-82 years) [1-3]. The retro peritoneum is reported to be the most frequent site of occurrence, while the frequency of adrenal gland nucleus is less than that found in children [4-6]. Therefore, we sought cases of neuroblastoma in Japanese adults using the following method. The search period was from 1983 to 2017, and the searches were conducted in MEDLINE and medicine central journals using the terms neuroblastoma, adult, and Japan; the searches excluded conference proceedings as well as cases with head, limbs, and bones as sites of tumor occurrence. As a result, we found 35 cases in 35 years in Japan, including our case [7-11], with two mediastinal, 16 retroperitoneal, 10 adrenal gland, and seven other tumors. In Japan, the population over 16 years old is estimated to be approximately 100 million a year; therefore, the incidence of adult neuroblastoma in Japan is at least one in 100 million people.

The 16 patients with primary adult onset retroperitoneal neuroblastoma in Japan, including our case, are shown in Table 2. The age of the patients ranged from 20 to 74 years (average, 40; seven male and nine female, showing a slightly greater frequency in women). Of these patients, seven had stage IV disease. Similarly to what was previously reported [7]. Stage IV disease is more common in adults than in children. This is thought to be because an epidemiological reason would be the rarity of neuroblastoma in adults, which makes it less suspect by physicians. Thus, the time to diagnosis is longer than that for children.

As a surgical method, in recent years, retroperitoneal access via the retroperitoneal mucosa is often performed to approach the kidney. The retroperitoneal access method does not require passing the pancreas or liver to secure the field of vision, and it is believed to reduce the risk of injury to intra-abdominal organs $[12,13]$. In addition, in this method, the vasculature entering from the dorsal side of the tumor can be processed early in surgery. In our case as well, it was possible to process the blood vessels from the dorsal side of the tumor using the retroperitoneal access method. In the case of primary retroperitoneal neuroblastoma, surgery using the retroperitoneal cavity for access was considered effective.

Regarding prognosis, the five-year survival rate of patients with stages III and IV disease is $28 \%$, and the median value when metastasis is recognized is reported to be $1.6 \%$ [1]. As shown in (Table 2) the survival rate of patients with stages III and IV disease is similarly low in cases of primary adult retroperitoneal neuroblastoma in Japan. In addition, although MYCN gene amplification is a poor prognostic factor for neuroblastoma, it is reported not to be a prognostic factor in adults compared to children, even in cases when the MYCN gene is not amplified and metastasis is not seen [14].

Thus, adult neuroblastoma is considered to have a poor prognosis because the standard treatment has not been established and the current situation demands referring to the guidelines for children for treatment. For this reason, in recent years, cases in which ICE therapy was effective for children have been reported [15] and as expected, cases of usage of ICE therapy in adults have been reported $[1,16]$ as in our case. However, adults, as in the case of our patient, may refuse chemotherapy due to the serious side effects of chemotherapy such as nausea and vomiting; therefore, treatment may not be performed adequately in line with the guidelines, which seems to be a problem.

\section{Conclusion}

The incidence of adult neuroblastoma in Japan is estimated to be approximately at least 1 in 100 million people. Because chemotherapy for adult neuroblastoma has strong side effects, the patient may refuse chemotherapy. As a result, treatment according to the guidelines may become difficult, which is a problem. As a surgical method, the retroperitoneal mirror was useful for exfoliation of the dorsal side of the tumor. In addition, even in adults with a non-amplified MYCN gene, prognosis is often poor and sufficient observation is necessary in the future.

\section{Conflict of Interest}

All authors report no conflict of interest.

\section{Reference}

1. Godkhindi VM (2016) Adult Neuroblastoma-Case Report and Literature Review. J Clin Diagn Res.

2. 2. Conter H, Gopalakrishnan V, Ravi V, Ater JL, Patel S, et al. (2014) Adult versus Pediatric Neuroblastoma: The M.D. Anderson Cancer Center Experience Sarcoma 2014: 1-6.

3. Hasegawa T, Hirose T, Ayala AG, Ito S, Tomaru U, et al. (2001) Adult neuroblastoma of the retroperitoneum and abdomen: clinicopathologic 
Citation: Watanabe S, Hara F, Kondo Y, Suzuki T, Yasui T, et al. (2018) A Case of Retroperitoneal Primary Neuroblastoma that Developed in Adulthood. J of Tumor Res \& Reports 3: 116.

distinction from primitive neuroectodermal tumor. Am J Surg Pathol 25: 918-924.

4. 4.Kaye JA, Warhol MJ, Kretschmar C, Landsberg L, lii EF (1986) Neuroblastoma in adults. Three case reports and a review of the literature. Cancer 58: 11491157.

5. Allan SG, Cornbleet MA, Carmichael J, Arnott SJ, Smyth JF (1986) Adult neuroblastoma. Report of three cases and review of the literature. Cancer 57:2419-2421.

6. Podda MG, Luksch R, Polastri D (2010) Neuroblastoma in patients over 12 years old: a 20-year experience at the Istituto Nazionale Tumori of Milan. Tumori 96:684-689.

7. Ichino M, Tsuruta T, Ogawa A, Ichikawa T, Ishii K, et al. (2001) A case of adult neuroblastoma arising in the retroperitoneal space. Nihon Hinyokika Gakkai Zasshi 92:632-635.

8. Tateishi U, Hasegawa T, Makimoto A, Moriyama N (2003) Adult neuroblastoma: radiologic and clinicopathologic features. J Comput Assist Tomogr 27: 321-326.

9. Tsuji A, Takayanagi M, Kasugai T, Sugimoto H, Shibahara H, et al. (2010) A case of adult neuroblastoma with fatal outcome. Nihon Shokakibyo Gakkai Zasshi 107: 1651-1660

10. Ohtaki Y, Ishii G, Hasegawa T, Nagai K (2011) Adult neuroblastoma arising in the superior mediastinum. Interact Cardiovasc Thorac Surg 13: 220-222.
11. Kurokawa S, Mizuno K, Nakane A, Moritoki Y, Nishio H, et al. (2016) Adrenal Neuroblastoma in an Adult: Effect of Radiotherapy on Local Progression after Surgical Removal. Case Rep Urol 16: 1-4.

12. Tanaka M, Ono Y, Matsuda T, Terachi T, Suzuki K, et al. (2009) Guidelines for urological laparoscopic surgery. Int J Urol 16: 115-125.

13. Nigri G, Rosman AS, Petrucciani N, Fancellu A, Pisano M, et al. (2013) Metaanalysis of trials comparing laparoscopic transperitoneal and retroperitoneal adrenalectomy Surgery 153: 111-119.

14. Kushner BH, Kramer K, Laquaglia MP, Modak S, Cheung NK, et al. (2003) Neuroblastoma in adolescents and adults: the Memorial Sloan-Kettering experience. Med Pediatric Oncol 41: 508-515.

15. Donfrancesco A, Jenkner A, Castellano A, llari I, Milano GM, et al (2004) Ifosfamide/carboplatin/etoposide (ICE) as front-line, topotecan/ cyclophosphamide as second-line and oral temozolomide as third-line treatment for advanced neuroblastoma over one year of age. Acta Paediatr Suppl 93: 6-11.

16. Miranda Soares PB, Quirino Filho S, DE Souza PW, Ferreti Bonan PR, Júnior $\mathrm{MH}$, et al. (2010) Neuroblastoma in an adult: case report. Rev Med Chil 138: 1131-1134. 